\title{
Diameter measurement of cylindrical products with displacement compensation along the optical axis
}

\author{
V. V. Rakhmanov, S. V. Dvoynishnikov, D. O. Semenov \\ Kutateladze Institute of Thermophysics SB RAS \\ Russian Federation
}

\begin{abstract}
The problem of diameter measurement of cylindrical products using the adapted triangulation method is considered. A method to improve the accuracy of measurement is proposed by determining the displacement of an object in the work area along the optical axis. The description of the layout of the laboratory installation and the results of applying the method for exemplary objects are given.
\end{abstract}

Keywords-Vision system, cylinder diameter measurement, triangulation method.

\section{INTRODUCTION}

$\mathrm{C}$ urrently, various industrial tools are widely used to allow contactless measurement of the diameter of cylindrical products in the production process directly on the transporter [1-4]. Shadow and diffractive optical direct scattering measurement methods provide the best measurement accuracy. Such methods allow achieving a diameter measurement error of the order of $0.01 \%$ of the measurement range [5], [6]. However, it is not always possible to provide measurements on direct light scattering. For measurements of the geometric dimensions of objects using backscattering, the measurement accuracy is already about $0.1 \%$ of the measurement range [7], [8]. The use of backscattering vision systems or laser profilometers to control the geometric parameters of products is possible for those applications where this error is considered satisfactory.

Using high-resolution digital vision systems, there are additional requirements for the stability of the measurement facility and lighting conditions. The use of inexpensive lenses with high spatial aberration introduces an additional error in the measurement of dimensions, since they do not allow you to get a true image of the controlled product [9].

Figure 1 shows the change in apparent size $d D=D^{\prime}-D^{\prime \prime}$ of object diameter $\mathrm{D}$ at a displacement along the optical axis by an amount $\mathrm{dX}$.

It is possible to reduce measurement error within the limited offset range $(\mathrm{dx} \sim 1-2 \mathrm{~mm})$ by using telecentric optics [10],
[11]. However, if the displacement along the optical axis exceeds these values, then the telecentric lens will also not allow you to compensate for the movement of the object. In addition, a telecentric lens has a high cost.

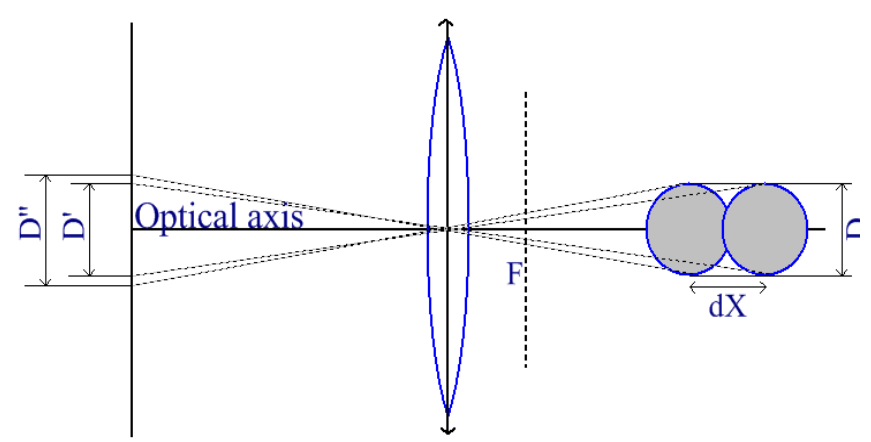

Fig. 1 Changing the size of the visible image when the displacement along the optical axis. $\mathrm{F}$ is the focal length of the lens, D is the diameter of the object, $\mathrm{D}^{\prime}$ and $\mathrm{D}$ " is the apparent diameter, $\mathrm{dX}$ is the displacement along the axis

Another way to reduce the measurement error is to use orthogonally located cameras [12], or to obtain a direct and reflected image from a mirror with a single camera [13]. From the orthogonal projection image, it is possible to calculate the displacement of the object along the optical axis and to introduce compensation for the scale of the direct image. A disadvantage of the method using two cameras is the need to use a second camera and to introduce an additional timing circuit to obtain a simultaneous image from both cameras. In industrial production, it is not always possible to place two cameras orthogonally. And when using mirrors, precise alignment of optical elements with temperature drift compensation and periodic maintenance of this system will be required. In addition, the complexity of image processing algorithms arises.

Methods of measuring geometric parameters based on triangulation methods of light section are known [14]. The method consists in illuminating the surface of the measured object with a light section and converting the light line observed at the photodetector into three-dimensional coordinates. The disadvantage of this method is that it has a high error when measuring cylindrical surfaces. Such a disadvantage is caused by a distortion of the measured 
geometric position of the points on the cylindrical surface due to different tilt angles of the light-scattering cylindrical surface.

The aim of this work is to develop a method for measuring the diameter of a cylindrical object, based on the triangulation method of light section, adapted for measuring a cylindrical surface.

\section{DESCRIPTION OF THE METHOD}

Figure 2 shows an optical scheme for measuring the cylinder diameter with to compensate for the displacement along the camera's optical axis. A laser light section of the smallest possible thickness is formed in the measuring zone to minimize the error in finding the distance.

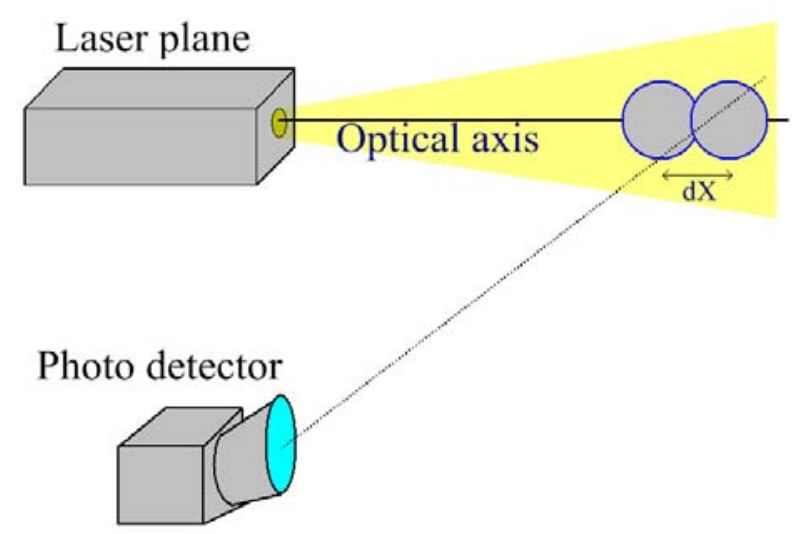

Fig. 2 Optical design of a cylinder diameter measuring system

The section is located to "cut" the cylinder across (fig. 3). Furthermore, the sectional plane axis is at an angle to the optical axis of the camera. Due to this angle, when moving an object along the optical axis, the image of the cross section moves on the photodetector. As a result, the image of a line curved by a cylindrical surface is analyzed (fig. 3 ).

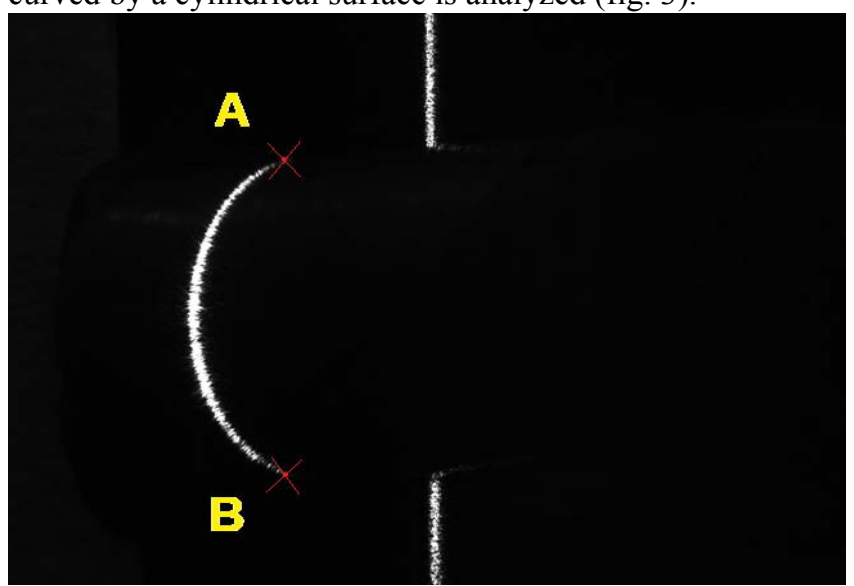

Fig. 3 Image of a cylinder in the light of a laser section. The coordinates of points $\mathrm{A}$ and $\mathrm{B}$ are analyzed

The cylinder diameter depends on the "visible" vertical line size on the photodetector and the compensation coefficient, taking into account the displacement of the object along the optical axis of the photodetector.

Let points $A$ and $B$ in the received image (Fig. 3) have coordinates $\left(X_{A}, Y_{A}\right)$ and $\left(X_{B}, Y_{B}\right)$, respectively. Then the cylinder diameter D will linearly depend on the vertical size of the line:

$$
D=M \square Y=M \square\left(Y_{B}-Y_{A}\right),
$$

where $\mathrm{M}$ is the conversion factor for the diameter of the object. The parameter $M$ has a quadratic dependence on the distance to the measured object L:

$M(L)=k \square L^{2}+m \square L+n$,

where $\mathrm{k}, \mathrm{m}$ and $\mathrm{n}$ are the coefficients calculated as a result of calibration. The distance $\mathrm{L}$ is calculated using the calibration function $\mathrm{F}$, which can be approximated by a polynomial of the third degree:

$$
L(X)=L\left(\frac{X_{A}+X_{B}}{2}\right)=a X^{3}+b X^{2}+c X+d,
$$

where $a, b, c$ and $d$ are the coefficients calculated as a result of calibration.

Thus, to measure the cylinder diameter, it is necessary to calculate two calibration functions: changing the visible vertical size from the distance to the object $\mathrm{M}(\mathrm{L})$ and the dependence of the distance to the object on the horizontal position of the light section line $\mathrm{L}(\mathrm{X})$.

\section{MAGE PROCESSING}

The proposed method of measuring the diameter of cylindrical objects requires precise determination of the position of points $\mathrm{A}$ and $\mathrm{B}$ on the received image (fig.3). It is obvious that the error of the method directly depends on the quality of the data processing algorithm for calculating the coordinates $\left(\mathrm{X}_{\mathrm{A}}, \mathrm{Y}_{\mathrm{A}}\right)$ and $\left(\mathrm{X}_{\mathrm{B}}, \mathrm{Y}_{\mathrm{B}}\right)$. In addition, the problem is complicated by the presence of local discontinuities in the observed light curve caused by the coherent nature of the light emitter used.

To solve this problem, the following approach was applied. First, a binarization operation based on the threshold transform [14] was applied to the image. Based on the analysis of the brightness histogram, the threshold value was selected:

Thresh $=\frac{M A X_{X, Y}(I(X, Y))+M I N_{X, Y}(I(X, Y))}{2}$,

where MAXX, Y (I (X, Y)) and MINX, Y (I (X, Y)) are the maximum and minimum intensities in the received image, respectively.

Next, a binary image $\operatorname{Ibin}(\mathrm{X}, \mathrm{Y})$ was constructed using a threshold transformation:

$I_{\text {bin }}(X, Y)=\left\{\begin{array}{l}1, \text { if } I(X, Y) \geq \text { Thresh } \\ 0, \text { if } I(X, Y)<\text { Thresh }\end{array}\right.$. 
Then function of dependence of position of center of mass of light spot $\mathrm{P}(\mathrm{Y})$ on binary image from vertical coordinate on image [15] was built:

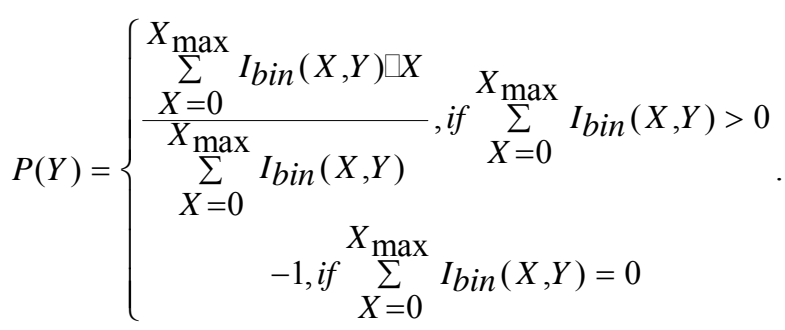

The next step of the algorithm is to filter the values of $\mathrm{P}(\mathrm{Y})$ corresponding to the reference surface: in Figure 3, they correspond to the line above point $\mathrm{A}$ and below point $\mathrm{B}$. To do so, select the value of the threshold $\mathrm{Tp}$ and equate all values above this threshold to invalid:

$$
P^{\prime}(Y)=\left\{\begin{array}{c}
-1, \text { if } \mathrm{P}(Y) \geq T p \\
P(Y), \text { if } \mathrm{P}(Y)<T p
\end{array}\right. \text {. }
$$

Then the coordinates of points A and B can be found from the following expressions:

$$
\begin{aligned}
& Y_{A}=\operatorname{MIN}_{P^{\prime}(Y)>0}(Y) . \\
& Y_{B}=\operatorname{MAX}_{P^{\prime}(Y)>0}(Y) . \\
& X_{A}=P^{\prime}\left(Y_{A}\right) . \\
& X_{B}=P^{\prime}\left(Y_{B}\right) .
\end{aligned}
$$

The resulting expressions allow the coordinates of points $\mathrm{A}$ and $\mathrm{B}$ to be calculated on an image received from a photodetector using both a coherent and non-coherent radiation source.

\section{LABORATORY INSTALLATION LAYOUT}

To test the operability of this method of diameter measurement, the laboratory installation layout was assembled (Fig. 4).

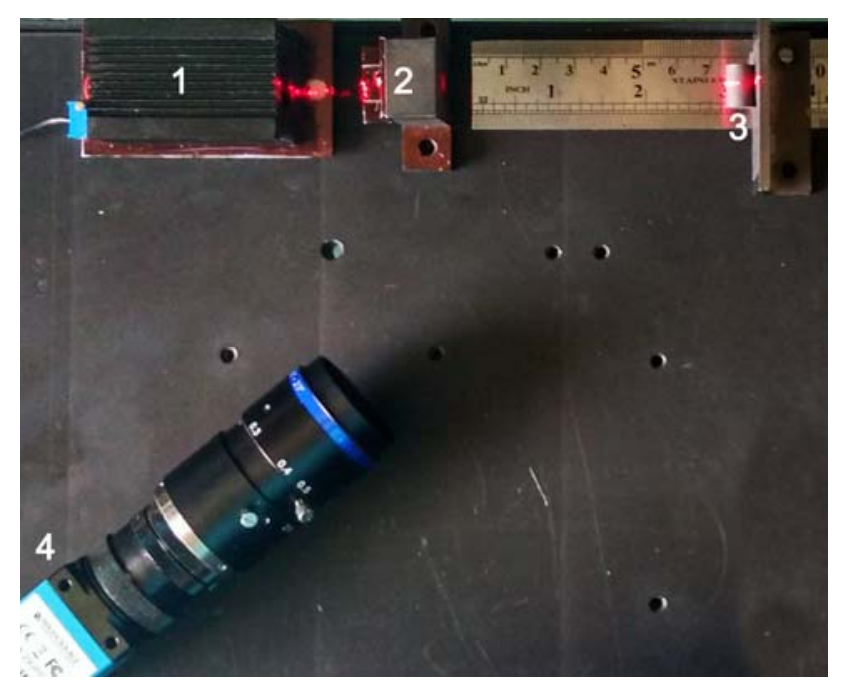

Fig. 4 Appearance of the model of the laboratory installation for measuring the diameter of the cylinder

A semiconductor laser emitter (1) $(650 \mathrm{~nm}, 5 \mathrm{~mW})$ with a constriction in the measuring zone. A cylindrical lens (2) was used to turn the beam into the plane of the laser section. Light plane intersects measured object (3) in half. The analyzed image arrived on the photodetector (4) (5 Mpx the DMK 23UP031 video camera and lens for cameras $5 \mathrm{Mpx}$ with variable focal distance of $15-8 \mathrm{~mm}$ ) located at an angle 45 degrees to optical axis of the emitter. All layout elements were located on a hard base to eliminate the effects of vibrations and temperature drifts. The maximum sharpness of the obtained image was achieved at a distance of $75 \mathrm{~mm}$ along the optical axis of the light emitter (in the constriction area). The zero mark on the optical axis is selected based on the overall dimensions of the optical system.

The object of measurement was a matte metal cylinder The cylinder diameter was measured using a mechanical micrometer with a measurement error of $1 \mu \mathrm{m}$. To reduce the error in measuring the diameter, 10 measurements were performed at the center and at the edges of the cylinder. and the arithmetic mean of these measurements was selected for the true size. The difference in the average cylinder diameters at the edges and center was no more than $30 \mu \mathrm{m}$. For the true diameter, the arithmetic mean value was selected from 10 measurements in the center, since measurements using the optical scheme were also performed in the center of the cylinder. The measured cylinder diameter was $8.196 \mathrm{~mm}$.

To calibrate the distance to the object depending on displacement of the visible light line in the received image, the cylinder was replaced with a reference plane (Fig. 5). The plane moved along optical axis of the emitter at distances from 60 to $100 \mathrm{~mm}$ with step of $10 \mathrm{~mm}$ with a positioning error of positioning about $\pm 0.5 \mathrm{~mm}$. 


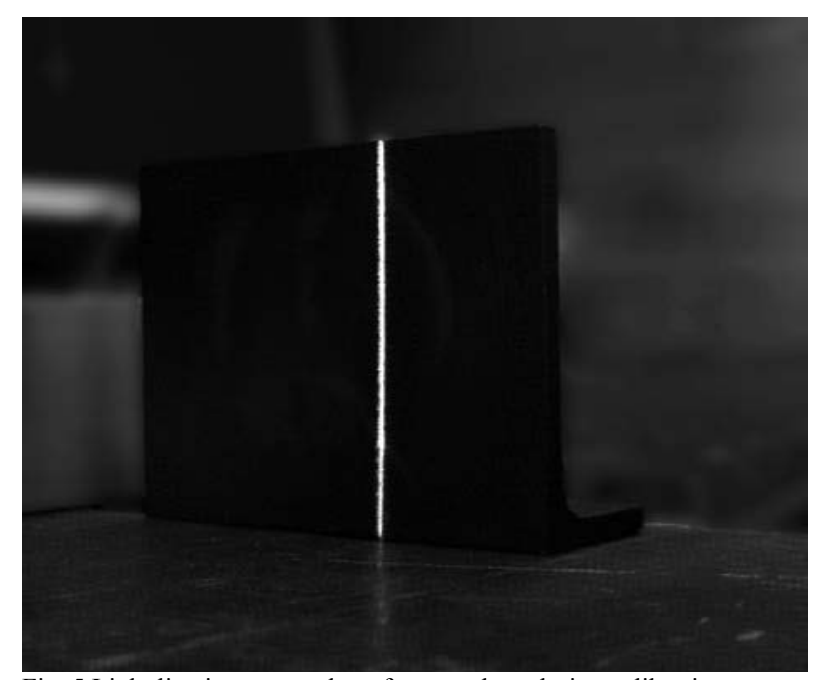

Fig. 5 Light line images on the reference plane during calibration

As a result of calibration, the coordinates $X$ of the light line in the image in pixels at 5 points along the axis are obtained. Figure 6 shows a graph of the distance L from the object from the $\mathrm{X}$ coordinate in the image.

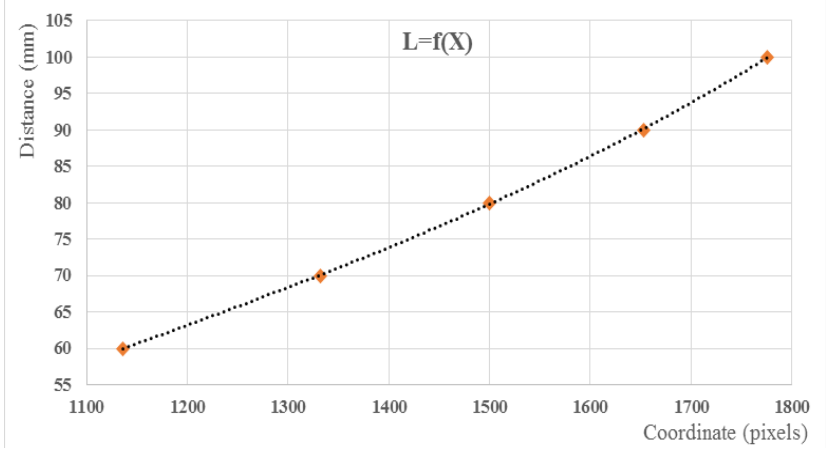

Fig. 6 Dependence of the distance to the object on the coordinate in the image

The calibration dependence of the distance to the object on the coordinate of the line of the laser light section in the camera image is described by a polynomial of the third degree:

$$
\begin{aligned}
& L=0.0000000260 \sqcap X^{3}-0.0000857961 * X^{2}, \\
& +0.1446171900 \square X-31.6625412629
\end{aligned},
$$

where $\mathrm{X}$ is the coordinate in pixels, $\mathrm{L}$ is the coordinate in millimeters. The interpolation error at nodal points was checked. The maximum interpolation deviation from these reference points is $0.4 \mathrm{~mm}$, which is a satisfactory result that is well consistent with the positioning error. This error can be improved with a more thorough calibration.

A linear calibration pattern (Fig. 7) for a microscope with a scale of $5 \mathrm{~mm}$ and a division price of $0.05 \mathrm{~mm}$ was used as a sample to determine the vertical axis scale factor as a function of the distance to the object. Full-scale error of the calibration pattern is not more than $0.015 \mathrm{~mm}$.

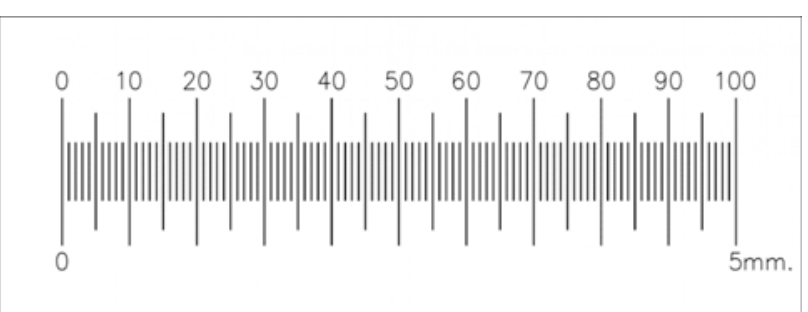

Fig. 7 Linear calibration pattern for the microscope.

This sample also moved in the range of distances from 60 to $100 \mathrm{~mm}$ in increments of $10 \mathrm{~mm}$ with a positioning error along the optical axis of $\pm 0.5 \mathrm{~mm}$. As a result of measurements, normalized values of the scaling factor depending on the distance were obtained (Fig. 8).

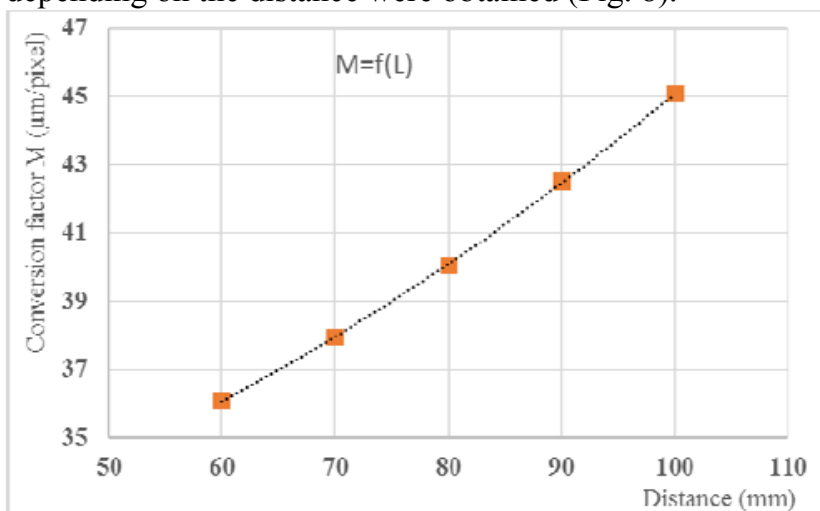

Fig. 8 The dependence of the scale factor $\mathrm{M}$ on the distance to the object

The dependence has a weak quadratic character (2) and is described by the following formula:

$M=0.0012 \square L^{2}+0.0322 \square L+29,8183 ，$

where $\mathrm{L}$ is the coordinate in millimeters, and $\mathrm{M}$ is the conversion factor of the object height in $\mu \mathrm{m} /$ pixel. The deviation of the measured data from the linear function does not exceed $0.2 \%$ of the values at the nodal points, which is consistent with the positioning error and the manufacturing error of the calibration pattern.

The diameter was measured by calculating the coordinates of the extreme points of the light laser section in the image of the cylinder. For this purpose threshold transformation of image was used and further location of extreme points on image of line on cylinder by method of center of mass in vicinity of most distant points $A$ and $B$ (fig.5).

From the coordinates of points $\mathrm{X}_{\mathrm{A}}$ and $\mathrm{X}_{\mathrm{B}}$, the arithmetic mean value of $\mathrm{X}$ was found, which was used to determine the distance to the object $\mathrm{L}$ by the formula (12). Then, the conversion factor $\mathrm{M}$ was found by formula (13) and the diameter value was calculated by the difference between YA and YB taking into account the coefficient M by formula (1). 


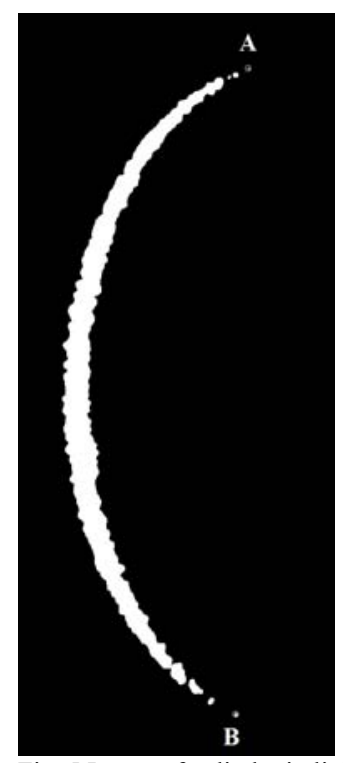

Fig. 5 Image of cylinder in light of laser section

For this layout, the expected diameter measurement errors were calculated. The pixel size of the selected camera is $2.2 \mathrm{x}$ $2.2 \mu \mathrm{m}$. Cylinder with a diameter of approximately $8.2 \mathrm{~mm}$ at the distance of $75 \mathrm{~mm}$ was visible on the image as 240 pixels or $0.528 \mathrm{~mm}$. Thus, the size reduction factor was 16 times. The coordinates of points A and B can be measured with an error of one pixel at best (sub-pixel interpolation was not used). However, due to the scaling factor, the measurement error will be about 35.2 microns. Therefore, the relative error of the diameter measurement (as the difference in the coordinates $\mathrm{Y}_{\mathrm{A}}$ and $\mathrm{Y}_{\mathrm{B}}$ ) can be about $70.4 \mu \mathrm{m}$.

It is possible to reduce this error by using a lens with a different focal length so that the image of the cylinder occupies the entire matrix area of the video camera. This will reduce the coefficient $M$ to 2 . Consequently, this will reduce the diameter error by eight times, to $8.8 \mu \mathrm{m}$.

\section{RESUlts}

In order to verify the operability of this method of compensating for the displacement of the object along the optical axis, two experiments were carried out. The first is the measurement of the cylinder at its movement in the range of 60 to $100 \mathrm{~mm}$. The second is the measurement of diameters of six different cylinders in a small range of movements of 75 $\mathrm{mm} \pm 1 \mathrm{~mm}$.

In the first case, a matte metal cylinder with a diameter of $8,196 \mathrm{~mm}$ was selected. Five cylinder diameter measurements were made. Each measurement was made according to the image processing after displacement of the cylinder along the optical axis in the range from 60 to $100 \mathrm{~mm}$ in increments of $10 \mathrm{~mm}$.

Table I presents the results of measuring the diameter of the cylinder in two variants: taking into account the adjustment to move along the optical axis $\mathrm{D}$ in the range $\mathrm{L}$ of $60-100 \mathrm{~mm}$ and without taking into account this amendment $\mathrm{Y}$ (only a scaling factor of pixels in millimeters in the middle of the range equal to 0.0389 ). Also for all measurements, deviations
$\Delta \mathrm{Y}$ and $\Delta \mathrm{D}$ from the actual cylinder diameter of $8.196 \mathrm{~mm}$ are presented.

From this table it can be seen that without taking into account the correction factor $\mathrm{M}$, the measurement of the diameter in the working area in the deviation range of \pm 20 $\mathrm{mm}$ has a high error. The measurement error is about $10 \%$.

\begin{tabular}{|l|c|c|c|c|}
\multicolumn{5}{|c}{ TABLE I } \\
\hline $\mathrm{L}, \mathrm{mm}$ & $\mathrm{Y}, \mathrm{mm}$ & $\Delta \mathrm{Y}, \mu \mathrm{m}$ & $\mathrm{D}, \mathrm{mm}$ & $\Delta \mathrm{D}, \mu \mathrm{m}$ \\
\hline 60 & 8.988 & -792 & 8.165 & 31 \\
\hline 70 & 8.562 & -366 & 8.189 & 7 \\
\hline 80 & 8.139 & 27 & 8.189 & 7 \\
\hline 90 & 7.746 & 450 & 8.272 & -76 \\
\hline 100 & 7.381 & 815 & 8.385 & -189 \\
\hline
\end{tabular}

It is also seen from the table that measurements at a distance of $100 \mathrm{~mm}$ give a high error in both cases. This is because the image was out of focus and was very blurry. That says that this method works indirectly with the output of the object from the measurement zone. If we remove the $100 \mathrm{~mm}$ point from the calculations, then the resulting standard deviation for the data with correction will be $76 \mu \mathrm{m}$. This is in good agreement with the calculated diameter measurement error for a given optical layout of a laboratory setup.

In the range of object displacements along the optical axis by $\pm 20 \mathrm{~mm}$, the measurement error was less than $1 \%$ of the cylinder diameter.

In the second experiment, the diameter of six different cylinders was measured at a distance of $75 \mathrm{~mm} \pm 1 \mathrm{~mm}$. This is a more realistic displacement of the cylinders when moving along a transport line. All cylinders had different reflective properties and various surface inhomogeneities.

The cylinders randomly moved over a given range of distances 10 times and each time their diameter was measured. For each cylinder, the resulting diameter was calculated as the arithmetic mean of 10 measurements. The true value of the cylinder diameter was taken as the arithmetic mean by the result of 10 measurements by a mechanical micrometer in the center of the cylinder.

The measurement results are presented in table II.

\begin{tabular}{|c|c|c|c|}
\hline TABLE II \\
\hline D real, mm & D mean, $\mathrm{mm}$ & $\Delta \mathrm{D}, \mu \mathrm{m}$ & $\mathrm{S}, \mu \mathrm{m}$ \\
\hline 8.235 & 8.225 & 10 & 19 \\
\hline 8.080 & 8.091 & -11 & 21 \\
\hline 8.153 & 8.145 & 8 & 22 \\
\hline 8.180 & 8.183 & -3 & 38 \\
\hline 8.224 & 8.220 & 4 & 22 \\
\hline 8.182 & 8.181 & 1 & 33 \\
\hline
\end{tabular}

The maximum error in measuring the average diameter was $11 \mu \mathrm{m}$.

Which is a good result for such a layout with a displacement of $\pm 1 \mathrm{~mm}$. In addition, the standard deviation of the diameter measurements for each cylinder was calculated from the results of 10 measurements. The standard deviation for all cylinders ranged from 19 to 38 microns. Which is in good agreement with the calculated errors for this optical design. 


\section{CONCLUSION}

The use of the adapted triangulation method has significantly reduced the error of determining the diameter of cylindrical objects at their displacements along the optical axis in the working area. In the layout of the laboratory setup was achieved error in determining the diameter of the cylinder 11 microns at displacement by $\pm 1 \mathrm{~mm}$. For displacements of \pm $20 \mathrm{~mm}$, an error in determining the diameter of $76 \mu \mathrm{m}$ was achieved.

Such a vision system provides accuracy acceptable for many tasks with a minimum of equipment costs. A more accurate calibration procedure and the selection of a more suitable lens allows us to hope for an additional reduction in measurement error.

\section{ACKNOWLEDGMENT}

This work was carried out under state contract with Kutateladze Institute of Thermophysics Siberian Branch of Russian Academia of Science.

\section{References}

[1] Dvoynishnikov S. V., Rakhmanov V. V., Meledin V. G., Kulikov D. V.,Anikin Yu. A., Kabardin I.K. Experimental Assessment of theApplicability of Laser Triangulators for Measurements of the Thicknessof Hot Rolled Product // Measurement Techniques. 2015, 57(12). -pp.1378-1385.

[2] Dvoinishnikov S.V., Anikin Y.A., Kabardin I.K., Kulikov D.V., MeledinV.G. An Optoelectronic Method of Contactless Measurement of theProfile of the Surface of Large Complexly Shaped Objects //Measurement Techniques. 2016. 59(1). - pp. 21-27.

[3] Dvoynishnikov S., Rakhmanov V. Power installations geometricalparameters optical control method steady against thermal indignations //EPJ Web of Conferences, № 82, 01035, 2015. - pp. 1-3.

[4] Kabardin I.K.,. Dvoynishnikov S.V, Meledin V.G., Naumov I.V. Thedistant diagnostics of transparent ice on wind turbine blades on the basisof total internal reflection // Journal of Engineering Thermophysics. 2016. № 4. pp.- 504-508.

[5] E. M. Fedorov, A. A. Koba Three-axis Laser Method for Measuring the Diameter of Cylindrical Objects // 2016 Dynamics of Systems, Mechanisms and Machines, Dynamics 2016, Omsk, November 15-17, 2016. - New York: IEEE, 2017 - pp. 1-4.

[6] Chugui Yu .V., Pavlov A. A. Peculiarities of cylinder diameter determination by diffraction // Proc. XVI IMEKO World Congress. Vienna, Austria, 2000. P. 169.

[7] Gerd-Joachim Deppe, Norbert Schönartz, Holger BrauerJörn Winkels Patent US 9,134,116 B2 - 2015.

[8] Lavrinov, D. S. / Analysis of Measurement System Accuracy Based on 2D Laser Triangulation Scanner When Measuring Soiled Pipe Thread Pitch and Height. Proceedings of the 5th International Conference on Industrial Engineering, ICIE 2019 - Volume II. Springer Verlag, 2020. pp. 521-529.
[9] Cross, R. (2019). Correcting camera perspective errors. Physics Education, 54(6), pp. 1-3.

[10] Gao, Yuan and Zhang, Guo Yu High Precision Machine Vision System for Outer Diameter Inspection Bearing //Key Engineering Materials, Vol. 552, 2013, pp. 313318.

[11] S. Zhang, B. Li, F. Ren and R. Dong, "High-Precision Measurement of Binocular Telecentric Vision System With Novel Calibration and Matching Methods," in IEEE Access, vol. 7, pp. 54682-54692, 2019.

[12]Z. Jia, W. Liu, and F. Wang, "Improved camera calibration method based on perpendicularity compensation for binocular stereo vision measurement system," Opt. Express 23, 15205-15223 (2015).

[13]Jiang W., Shimizu M., Okutomi M. (2010) SingleCamera Multi-baseline Stereo Using Fish-Eye Lens and Mirrors. In: Zha H., Taniguchi R., Maybank S. (eds) Computer Vision - ACCV 2009. ACCV 2009. Lecture Notes in Computer Science, vol 5995. Springer, Berlin, Heidelberg. pp. 347-358,

[14] Quanli Liu, Jia Li and Peifen Chen, "Measurement of cylinder diameter based on computer vision system," IET International Conference on Information Science and Control Engineering 2012 (ICISCE 2012), Shenzhen, 2012, pp. 1-4.

[15] Rafael C. Gonzalez (2008). Digital Image Processing. Prentice Hall.

[16] Oppenheim, Alan V.; Schafer, Ronald W. (2001). Discrete-Time Signal Processing. Pearson. 